\title{
Further solutions of critical ABF RSOS models
}

\author{
Yu-kui Zhou円' \\ Mathematics Department, University of Melbourne, \\ Parkville, Victoria 3052, Australia \\ and \\ Department of Mathematics, The Australian National University \\ Canberra, ACT 0200, Australia
}

\begin{abstract}
The restricted SOS model of Andrews, Baxter and Forrester has been studied. The finite size corrections to the eigenvalue spectra of the transfer matrix of the model with a more general crossing parameter have been calculated. Therefore the conformal weights and the central charges of the non-unitary or unitary minimal conformal field have been extracted from the finite size corrections.
\end{abstract}

hep-th/9504107

revised version

\section{Introduction}

The ABF restricted solid-on-solid (RSOS) model has been found by Andrews, Baxter and Forrester $(\mathrm{ABF})$ in 1984 [1]. It has been well known that the model provides realizations of unitary minimal conformal field theories [2, 3, 国. This has been further confirmed by studying the finite-size corrections to the ground state energy [5]-[11] (also see [12]- [22] for related works). Among these works, much effort has been focused on the ABF model corresponding to the unitary minimal conformal field theories. By contrast, the finite-size corrections to the transfer matrix of the ABF model corresponding to non-unitary minimal conformal field theories have received no attention.

\footnotetext{
${ }^{1}$ Email: zhouy@maths.anu.edu.au

${ }^{2}$ On leave of absence from Institute of Modern Physics, Northwest University, Xian 710069, China

${ }^{3}$ Postal address.
} 
The local height probabilities of the ABF model with a crossing parameter $\lambda=k \pi / h$, where two relatively prime positive integers satisfy $k<h$, have been calculated in [23]. In this paper, with the same motivation, we repeat the consideration of the finite-size correction calculation of the $\mathrm{ABF}$ model with $\lambda=k \pi / h$. In general the model will no longer be physical as there will be some negative face weights. Nevertheless, the non-unitary minimal conformal field theories [4] can be realized as the critical continuum of the ABF RSOS model with the crossing parameter $\lambda=k \pi / h$. The model is therefore of independent interest for this feature.

In [20] an analytic method has been presented to find the finite-size corrections involving the central charges for the six-vertex model with a twisted boundary condition. The method has been successfully applied to the other models (see [27 for example). In these works only the central charges have been obtained. In fact the central charge and conformal weights together could appear in the finite-size corrections to the eigenvalue spectra of the transfer matrix. In this paper, following the calculation presented in [20], we find the finite-size corrections to the eigenvalue spectra of transfer matrices of the critical ABF RSOS model. From the corrections both the central charges and the conformal weights of non-unitary minimal conformal field theories are extracted. This generalizes the method presented in 20] to find the conformal weights of the ABF SOS model.

We first review the ABF RSOS model and the Bethe ansatz solutions of transfer matrices briefly in section 1.1. In section 2 we find an integral nonlinear equation and express the finite-size corrections in terms of the solution of the nonlinear equation. Then the effective central charges including the conformal weights are extracted from the finite-size corrections. A brief discussion is presented in the final section.

\subsection{Models and Bethe ansatz solutions}

The ABF RSOS model can be given by Baxter's SOS model, which was introduced in order to solve the eight-vertex model with the $R$-matrix

where

$$
R(u)=\left(\begin{array}{cccc}
a(u) & 0 & 0 & d(u) \\
0 & b(u) & c(u) & 0 \\
0 & c(u) & b(u) & 0 \\
d(u) & 0 & 0 & a(u)
\end{array}\right)
$$

$$
\begin{array}{ll}
a(u)=\Theta(\lambda) \Theta(u) H(u+\lambda), & b(u)=\Theta(\lambda) H(u) \Theta(u+\lambda), \\
c(u)=H(\lambda) \Theta(u) \Theta(u+\lambda), & d(u)=H(\lambda) H(u) H(u+\lambda) .
\end{array}
$$

The $R$-matrix satisfies the Yang-Baxter equation [24, 25]

$$
R^{12}(u) R^{13}(u+v) R^{23}(v)=R^{23}(v) R^{13}(u+v) R^{12}(u) .
$$

Baxter has shown in [25] that the eight-vertex model can be transferred into the SOS model, which is defined by the following face weights

$$
W(\ell \pm 1, \ell \pm 2, \ell \pm 1, \ell \mid u)=\frac{h(u+\lambda)}{h(\lambda)}
$$




$$
\begin{aligned}
W(\ell \mp 1, \ell, \ell \pm 1, \ell \mid u) & =\frac{h(\xi+\ell \lambda \pm \lambda) h(u)}{h(\xi+\ell \lambda) h(\lambda)} \\
W(\ell \pm 1, \ell, \ell \pm 1, \ell \mid u) & =\frac{h(\xi+\ell \lambda \mp u)}{h(\xi+\ell \lambda)}
\end{aligned}
$$

where the height $\ell \in \mathbb{Z}$ and $\xi$ is an independent parameter. The crossing parameter is $\lambda$ and the spectral parameter is $u$. The function $h(u)$ is given by

$$
h(u)=\Theta(0) H(u) \Theta(u) .
$$

The face weights satisfy the following Yang-Baxter equation

$$
\begin{array}{rl}
\sum_{g} & W(a, b, g, f \mid u) W(f, g, d, e \mid v) W(g, b, c, d \mid v-u) \\
& =\sum_{g} W(f, a, g, e \mid v-u) W(a, b, c, g \mid v) W(g, c, d, e \mid u)
\end{array}
$$

for any integers $a, b, c, d, e, f$. Therefore the SOS model is an integrable system. Suppose that $\boldsymbol{l}$ and $\boldsymbol{m}$ are allowed spin configurations of two consecutive rows of an $N$ (even) column lattice with periodic boundary conditions $l_{N+1}=l_{1}, m_{N+1}=m_{1}$. The elements of the rowto-row transfer matrix $\boldsymbol{T}$ of the SOS model are defined by

$$
\langle\boldsymbol{l}|\mathbf{T}(u)| \boldsymbol{m}\rangle=\prod_{j=1}^{N} W\left(l_{j}, l_{j+1}, m_{j+1}, m_{j} \mid u\right) .
$$

We recall the eigenvalues of the transfer matrix $\boldsymbol{T}$ given in [25] (also see [26] for algebraic Bethe ansatz),

$$
T(u)=e^{\mathrm{i} s \lambda} h^{N}\left(u+\frac{1}{2} \lambda\right) \frac{q(u-\lambda)}{q(u)}+e^{-\mathrm{i} s \lambda} h^{N}\left(u-\frac{1}{2} \lambda\right) \frac{q(u+\lambda)}{q(u)}
$$

where $q(u)$ is defined by

$$
q(u)=\prod_{j=1}^{N / 2} h\left(u-u_{j}\right) .
$$

These parameters $u_{1}, u_{2}, \cdots, u_{N / 2}$ are determined by the Bethe ansatz equations,

$$
p\left(u_{j}\right)=-1, \quad j=1,2, \cdots, N / 2
$$

where the function is given by

$$
p(u):=e^{-2 \mathrm{i} s \lambda} \frac{h^{N}\left(u-\frac{1}{2} \lambda\right) q(u+\lambda)}{h^{N}\left(u+\frac{1}{2} \lambda\right) q(u-\lambda)} .
$$

The ABF RSOS model is specialized by setting

$$
\lambda=k \pi / h \text { and } \xi=0
$$

where $k$ and $h$ are relatively prime integers $(h>k>0)$ and $s=1,2, \cdots, h-1$. With this condition (1.12) the face weights still satisfy the Yang-Baxter equation. The row-to-row transfer matrix $\boldsymbol{T}(u)$ forms the commuting family

$$
[\boldsymbol{T}(u), \boldsymbol{T}(v)]=0 .
$$

Therefore the model is integrable. The Bethe ansatz solutions (1.8) and (1.10) with the restriction (1.12) are the eigenvalues and the Bethe ansatz equations of the transfer matrix of the RSOS model [7, 8]. 


\section{Finite-size corrections}

We consider the corresponding critical ABF RSOS model, which can be obtained by taking the zero elliptic nome $p=0$. The elliptic function $h(u)$ reduces to the trigonometric function

$$
h(u)=\sin (u)
$$

if $p \rightarrow 0$. The eigenvalues (1.8) and the Bethe ansatz equations (1.10) are still correct for the critical RSOS model if the function $h(u)$ is replaced with (2.1).

Let us introduce the new spectral variable $v=i u$. It is very helpful to notice that the eigenvalue spectra (1.8) and the Bethe ansatz equations (1.10) are the same as those of the transfer matrix of the six vertex model with a twisted boundary condition [20]. They therefore can be treated similarly. The functions have to be restricted in some analyticity domain since all functions are i $\pi$-periodic. It has been shown in [20] that the following functions are analytic and non-zero (ANZ)

$$
\begin{array}{ccll}
h(v) & \text { ANZ } & \text { in } & 0<\Im m(v)<\pi \\
q(v) & \text { ANZ } & \text { in } & -\pi<\Im m(v)<0 \\
T(v) & \text { ANZ } & \text { in } & -\lambda / 2 \leq \Im m(v) \leq \lambda / 2,
\end{array}
$$

and, respectively, the functions $q$ and $p$ satisfy

$$
\bar{q}(v)=q(\bar{v}) \text { and } \bar{p}(v)=1 / p(v) \text {. }
$$

Because of $\mathrm{i} \pi$-periodic functions of face weights we can take $k<h / 2$. Note that (2.2) has restricted the model to stay on the critical line of regime III/IV .

\subsection{Nonlinear integral equation}

Following [20], let us introduce new functions

$$
\begin{gathered}
\alpha(x):=1 / p(x-\mathrm{i} \lambda / 2)=\left[\tanh \frac{\pi x}{2 \lambda}\right]^{N} a(x) \\
\mathcal{A}(x):=1+\alpha(x)
\end{gathered}
$$

The variable $x$ may be regarded as real.t The method presented in [20] is to derive a set of relations about functions $a$ and $q$ and these relations lead to a nonlinear integral equation, in turn, the nonlinear integral equation ensures that the finite-size corrections to the eigenvalue spectra of the transfer matrix can be solved through dilogarithmic functions.

The involved functions are ANZ in the strips (2.2) and are exponentials in asymptotic behaviour. The second logarithmic derivatives of the functions can be Fourier transformed,

$$
\begin{aligned}
f(k) & =\frac{1}{2 \pi} \int_{-\infty}^{\infty}[\ln f(x)]^{\prime \prime} e^{-\mathrm{i} k x} d x \\
{[\ln f(x)]^{\prime \prime} } & =\int_{-\infty}^{\infty} f(k) e^{\mathrm{i} k x} d k
\end{aligned}
$$

\footnotetext{
${ }^{4}$ Sometimes it is convenient to work with values of $x$ in the upper half plane close to the real axis for avoiding singularities which might otherwise occur.
} 
where the integration path in $x$-plane has to lie in the analyticity strip and the real part of the variable of integration goes from $-\infty$ to $\infty$. By Cauchy's theorem all other details of the path are irrelevant for $f(k)$.

We now derive a set of relations about functions $a$ and $q$. Applying the Fourier transform to the definition (2.4) of $a(x)$

$$
a(x)=e^{2 \mathrm{i} s \lambda}\left[\operatorname{coth} \frac{\pi x}{2 \lambda}\right]^{N} \frac{h^{N}(x) q(x-3 \mathrm{i} \lambda / 2)}{h^{N}(x-\mathrm{i} \lambda+\mathrm{i} \pi) q(x+\mathrm{i} \lambda / 2-\mathrm{i} \pi)}
$$

where all arguments of the functions $q$ and $h$ have been reduced to the analyticity strips (2.2) because of the $\pi$ i-periodicity, then yields

$$
a(k)=-\frac{N k}{1+e^{-\lambda k}}+\frac{N k\left(1-e^{(\lambda-\pi) k}\right)}{1-e^{-\pi k}}+\left(e^{\frac{3}{2} \lambda k}-e^{\left(\pi-\frac{1}{2} \lambda\right) k}\right) q(k) .
$$

To solve $a$ and $q$ we need another relation, which is introduced by an auxiliary function

$$
h_{\mathrm{a}}(v):=\frac{1+p(v)}{q(v)} .
$$

It is ANZ in the strip $-\lambda / 2<\Im m(v) \leq \lambda / 2$. To apply Cauchy's theorem to the Fourier transform of the second logarithmic derivative of $h_{a}$ we rewrite $h_{\mathrm{a}}(v)$ in the following two different forms such that the arguments of $q$ stands in the analyticity strip (2.2)

$$
\begin{aligned}
h_{\mathrm{a}}(x-\mathrm{i} \lambda / 2) & =\left[\operatorname{coth} \frac{\pi x}{2 \lambda}\right]^{N} \frac{\mathcal{A}(x)}{q(x-\mathrm{i} \lambda / 2) a(x)} \\
h_{\mathrm{a}}(x+\mathrm{i} \lambda / 2) & =\frac{\overline{\mathcal{A}}(x)}{q(x+\mathrm{i} \lambda / 2-\mathrm{i} \pi)} .
\end{aligned}
$$

Applying the Fourier transform to (2.9), it follows that

$$
\begin{aligned}
e^{\lambda k / 2} h_{\mathrm{a}}(k) & =-\frac{N k}{1+e^{-\lambda k}}-e^{\lambda k / 2} q(k)+\mathcal{A}(k)-a(k) \\
e^{-\lambda k / 2} h_{\mathrm{a}}(k) & =\overline{\mathcal{A}}(k)-e^{(\pi-\lambda / 2) k} q(k) .
\end{aligned}
$$

Then they are equated yielding

$$
q(k)\left(e^{(\pi+\lambda / 2) k}-e^{\lambda k / 2}\right)=\frac{N k}{1+e^{-\lambda k}}+a(k)+e^{\lambda k} \overline{\mathcal{A}}(k)-\mathcal{A}(k) .
$$

The equations (2.7) and (2.10) together determine the functions $a(k)$ and $q(k)$,

$$
\begin{aligned}
a(k)= & \frac{\sinh \left(\frac{1}{2} \pi k-\lambda k\right)}{2 \cosh \left(\frac{1}{2} \lambda k\right) \sinh \frac{1}{2}(\pi k-\lambda k)}\left(\mathcal{A}(k)-e^{(\lambda-\epsilon) k} \overline{\mathcal{A}}(k)\right) \\
q(k)= & \frac{N k e^{-\pi k / 2}}{4 \sinh \left(\frac{1}{2} \pi k\right) \cosh \left(\frac{1}{2} \lambda k\right)} \\
& -\frac{e^{-(\pi+\lambda) k / 2}}{4 \cosh \left(\frac{1}{2} \lambda k\right) \sinh \frac{1}{2}(\pi k-\lambda k)}\left(\mathcal{A}(k)-e^{\lambda k} \overline{\mathcal{A}}(k)\right)
\end{aligned}
$$

where an infinitesimal positive $\epsilon$ has been introduced for the imaginary part of the argument of $x$. Transforming back to the variable $x$

$$
[\ln a(x)]^{\prime \prime}=\int_{-\infty}^{\infty}\left(K(y)[\ln \mathcal{A}]^{\prime \prime}(x-y)-K(y+\mathrm{i} \epsilon-\mathrm{i} \lambda)[\ln \overline{\mathcal{A}}]^{\prime \prime}(x-y)\right) d y
$$


where the kernel function

$$
K(x):=\frac{1}{2 \pi} \int_{-\infty}^{\infty} \frac{\sinh \left(\frac{1}{2} \pi-\lambda\right) k}{2 \cosh \left(\frac{1}{2} \lambda k\right) \sinh \frac{1}{2}(\pi-\lambda) k} e^{i k x} d k
$$

satisfies

$$
\bar{K}(x)=K(-\bar{x}), \quad K(x)=K(-x) .
$$

The equation (2.12) is derived based on the essential ANZ property of the Bethe ansatz solution (1.8). Low-lying excitations have the same bulk behavior as the ground state. The only difference has been shown in 9 to lie in the fact that the eigenvalue functions now possess a finite number of zeros in the analyticity strip, which were free of zeros in the ground state. However, it is always possible to take an ANZ area in the analyticity strip where Cauchy's theorem can be applied [11. Therefore the equation (2.12) still works for the excited states if we change the integration path in the ANZ area. Integrating (2.12) twice we obtain a nonlinear integral equation

$$
\begin{gathered}
\ln a(x)=\int_{-\infty}^{\infty}(K(y) \ln \mathcal{A}(x-y)-K(y+\mathrm{i} \epsilon-\mathrm{i} \lambda) \ln \overline{\mathcal{A}}(x-y)) d y \\
+C+D x
\end{gathered}
$$

where the integral constant $D=0$ because all terms remain finite for $x \rightarrow \infty$ and another integral constant $C$ heavily dependent on the branch choice of $\ln a(x)$,

$$
\begin{aligned}
C & =\ln a(\infty)-\int_{-\infty}^{\infty} K(y) d y(\ln \mathcal{A}(\infty)-\ln \overline{\mathcal{A}}(\infty)) \\
& =\ln \left(\omega^{2} e^{2 \mathrm{i} s \lambda}\right)-\frac{\frac{1}{2} \pi-\lambda}{\pi-\lambda}\left(\ln \left(1+\omega^{2} e^{2 \mathrm{i} s \lambda}\right)-\ln \left(1+\omega^{-2} e^{-2 \mathrm{i} s \lambda}\right)\right) \\
& =\frac{\mathrm{i} \pi \phi}{\pi-\lambda}
\end{aligned}
$$

where the phase factor $\phi$ has been introduced by

$$
\phi=s \lambda-\mathrm{i} \ln \omega, \quad \omega^{2}=1 .
$$

Here we have taken a more general choice of branches for $\ln a(x)$, or $\ln a(\infty)=2 \ln \left(\omega e^{\mathrm{i} s \lambda}\right)$. The case $\omega=1$ has been studied in [20], which corresponds to the ground state. For the excited states we have chosen the other branches with $\omega \neq 1$ or take

$$
\omega=e^{\mathrm{i}(t-s) \pi}
$$

with the integers $s, t$. From the definition (2.4) it follows that $a$ goes to $a e^{-4 i s \lambda}$ under the change $s \rightarrow h-s$. So the same symmetry should be imposed on the equation (2.15), or the phase factor $\phi$ must go to $-\phi$ under this change. It follows that the phase factor $\phi$ will go to $-\phi$ if changing $s \rightarrow h-s$ and $t \rightarrow h-k-t$. Similar to the exponent $s$, suppose that $t$ is positive. According to $s=1,2, \cdots, h-1$ we therefore take $t=1,2, \cdots, h-k-1$. Recalling the definition (2.4) we arrived at the nonlinear integral equation for $\alpha$

$$
\begin{aligned}
\ln \alpha(x)= & N \ln \tanh \frac{\pi x}{2 \lambda}+\frac{\mathrm{i} \pi \phi}{\pi-\lambda} \\
& +\int_{-\infty}^{\infty}(K(y) \ln \mathcal{A}(x-y)-K(y+\mathrm{i} \epsilon-\mathrm{i} \lambda) \ln \overline{\mathcal{A}}(x-y)) d y .
\end{aligned}
$$


This equation is exact for all finite system size and for both the ground state and the excited states.

\subsection{Scaling limits}

To obtain the finite-size corrections to the eigenvalue spectra of the transfer matrix we observe the following scaling behaviour

$$
\lim _{N \rightarrow \infty}\left(\tanh \left[\frac{\pi}{2 \lambda}\left( \pm \frac{\lambda}{\pi}(x+\ln N)\right)\right]\right)^{N}=\exp \left(-2 e^{-x}\right)
$$

in thermodynamic limit $N \rightarrow \infty$. The function $\alpha$ scale similarly,

$$
\begin{array}{cc}
a_{ \pm}(x):=\lim _{N \rightarrow \infty} \alpha\left( \pm \frac{\lambda}{\pi}(x+\ln N)\right), & l a_{ \pm}(x):=\ln a_{ \pm}(x) \\
A_{ \pm}(x):=\lim _{N \rightarrow \infty} \mathcal{A}\left( \pm \frac{\lambda}{\pi}(x+\ln N)\right)=1+a_{ \pm}(x), \quad l A_{ \pm}(x):=\ln A_{ \pm}(x) .
\end{array}
$$

In the scaling limit regimes the nonlinear integral equation (2.19) becomes

$$
\begin{aligned}
l a_{ \pm}(x)= & -2 e^{-x}+\int_{-\infty}^{\infty} K_{1}(x-y) l A_{ \pm}(y) d u \\
& -\int_{-\infty}^{\infty} K_{2}(x-y) \overline{l A}_{ \pm}(y) d u+\frac{\mathrm{i} \pi \phi}{\pi-\lambda} \\
\overline{l a}_{ \pm}(x)= & -2 e^{-x}+\int_{-\infty}^{\infty} \bar{K}_{1}(x-y) \overline{l A}_{ \pm}(y) d u \\
& -\int_{-\infty}^{\infty} \bar{K}_{2}(x-y) l A_{ \pm}(y) d u-\frac{\mathrm{i} \pi \phi}{\pi-\lambda}
\end{aligned}
$$

where $K_{1,2}(x)$ are defined by

$$
\begin{aligned}
K_{1}(x) & :=\frac{\lambda}{\pi} K\left(\frac{\lambda}{\pi} x\right) \\
K_{2}(x) & :=\frac{\lambda}{\pi} K\left(\frac{\lambda}{\pi} x \pm \mathrm{i}(\epsilon-\lambda)\right) .
\end{aligned}
$$

Let us now turn to the eigenvalues $T$ given by (1.8). Its finite-size corrections can be derived from

$$
T(x-\mathrm{i} \lambda / 2)=h^{N}(x-\mathrm{i} \lambda) \frac{q(x+\mathrm{i} \lambda / 2-\mathrm{i} \pi)}{q(x-\mathrm{i} \lambda / 2)} \mathcal{A}(x) e^{-s \mathrm{i} \lambda} .
$$

Applying the Fourier transform to the ratio of the $q$-functions and taking (2.11) into account we have

$$
\begin{aligned}
& \ln \frac{q(x+\mathrm{i} \lambda / 2-\mathrm{i} \pi)}{q(x-\mathrm{i} \lambda / 2)}=-N \int_{-\infty}^{\infty} \frac{\sinh \frac{1}{2}(\pi-\lambda) k}{2 k \sinh \frac{1}{2}(\pi k) \cosh \frac{1}{2}(\lambda k)} e^{\mathrm{i} k x} d y \\
&+\frac{\mathrm{i}}{2 \lambda} \int_{-\infty}^{\infty} \frac{\ln \mathcal{A}(x-y)}{\sinh \frac{\pi}{\lambda}(y-\mathrm{i} \epsilon)} d y+\frac{\mathrm{i}}{2 \lambda} \int_{-\infty}^{\infty} \frac{\ln \overline{\mathcal{A}}(x-y)}{\sinh \frac{\pi}{\lambda}(y+\mathrm{i} \epsilon)} d y+f_{c}
\end{aligned}
$$


where $f_{c}$ is an integration constant. Therefore the finite-size corrections to the eigenvalue can be expressed as

$$
\begin{aligned}
\ln T(x-\mathrm{i} \lambda / 2)= & f_{c}+N \ln h(x-\mathrm{i} \lambda)-N \int_{-\infty}^{\infty} \frac{\sinh \frac{1}{2}(\pi-\lambda) k \sinh (x k)}{2 k \sinh \frac{1}{2}(\pi k) \cosh \frac{1}{2}(\lambda k)} d k \\
& +\frac{\mathrm{i}}{\lambda} \int_{-\infty}^{\infty} \frac{\Re e \ln \mathcal{A}(y)}{\sin \frac{\pi}{\lambda}(x-y+\mathrm{i} \epsilon)} d y+o\left(\frac{1}{N}\right) .
\end{aligned}
$$

The scaling limit of the corrections can be done by splitting the integral into two parts, then inserting the variable of integration $y$ by $\pm \frac{\lambda}{\pi}(y+\ln N)$ and using the scaling functions (2.22), we obtain

$$
\begin{aligned}
& \ln T(x-\mathrm{i} \lambda / 2) \\
& =-N f(x-\mathrm{i} \lambda / 2)-\frac{2 \mathrm{i}}{\pi N} e^{\frac{\pi}{\lambda} x} \int_{-\infty}^{\infty} \Re e l A_{+}(y) e^{-y} d y \\
& +\frac{2 \mathrm{i}}{\pi N} e^{-\frac{\pi}{\lambda} x} \int_{-\infty}^{\infty} \Re e l A_{-}(y) e^{-y} d y+o\left(\frac{1}{N}\right) \\
& =-N f(x-\mathrm{i} \lambda / 2)-\frac{\pi \mathrm{i}}{6 N} \sinh \frac{\pi x}{\lambda}\left(\frac{24}{\pi^{2}} \int_{-\infty}^{\infty} \Re e l A_{ \pm}(y) e^{-y} d y\right)+o\left(\frac{1}{N}\right)
\end{aligned}
$$

where the bulk behavior is entirely expressed by the first term and second term is the finitesize corrections. The integration constant $f_{c}$ is chosen so that $f(x-\mathrm{i} \lambda / 2)$ is exactly the bulk energy, which can be derived from the inversion relation of the face weights [7, 23]. Here we are only interested in the finite-size correction terms which include the conformal spectra.

\subsection{Conformal spectra}

The conformal spectra can be extracted from the finite-size corrections of the transfer matrix. The integral in the finite-size correction term in (2.28) can be calculated by considering the expression

$$
\begin{aligned}
& \int_{-\infty}^{\infty}\left(\left[l a_{ \pm}(x)\right]^{\prime} l A_{ \pm}(x)-l a_{ \pm}(x)\left[l A_{ \pm}(x)\right]^{\prime}\right) d x \\
& \quad+\int_{-\infty}^{\infty}\left(\left[\overline{l a}_{ \pm}(x)\right]^{\prime} \overline{l A}_{ \pm}(x)-\overline{l a}_{ \pm}(x)\left[\overline{l A}_{ \pm}(x)\right]^{\prime}\right) d x \\
& =2 \int_{-\infty}^{\infty} e^{-x}\left(l A_{ \pm}(x)+\left[l A_{ \pm}(x)\right]^{\prime}\right) d x \\
& \quad+2 \int_{-\infty}^{\infty} e^{-x}\left(\overline{l A}_{ \pm}(x)+\left[\overline{l A}_{ \pm}(x)\right]^{\prime}\right) d x \\
& \quad-\frac{\pi \mathrm{i} \phi}{\pi-\lambda} \int_{-\infty}^{\infty}\left(\left[l A_{ \pm}(x)\right]^{\prime}-\left[\overline{l A}_{ \pm}(x)\right]^{\prime}\right) d x
\end{aligned}
$$

The right hand side is derived by using the nonlinear integral equation (2.23). The left hand side can be calculated after changing the variable $x$ to $a$ and $\bar{a}$ and using the dilogarithmic function

$$
L(x)=\int_{0}^{x}\left(\frac{\ln (1+y)}{y}-\frac{\ln y}{1+y}\right] d y
$$


Then we are able to derive

$$
\begin{aligned}
& \frac{24}{\pi^{2}} \int_{-\infty}^{\infty} e^{-x} \Re e l A_{ \pm}(x) d x \\
& =\frac{3}{\pi^{2}}\left(L\left(\omega^{2} e^{2 i s \lambda}\right)+L\left(\omega^{-2} e^{-2 i s \lambda}\right)-\frac{2 \pi \phi^{2}}{\pi-\lambda}\right)
\end{aligned}
$$

where the asymptotics of $a_{ \pm}(\infty)=\left(\omega e^{\mathrm{i} s \lambda}\right)^{2}, \bar{a}_{ \pm}(\infty)=\left(\omega e^{\mathrm{i} s \lambda}\right)^{-2}$ and $a_{ \pm}(-\infty)=\bar{a}_{ \pm}(-\infty)=$ 0 have been read off from (2.23). Finally using the well known identity

$$
L(z)+L(1 / z)=\frac{\pi^{2}}{3}
$$

the finite-size corrections in (2.28) are given by the explicit expression

$$
\ln T(x-\mathrm{i} \lambda / 2)=-N f(x-\mathrm{i} \lambda / 2)-\frac{\pi \mathrm{i}}{6 N}(c-24 \Delta) \sinh \frac{\pi x}{\lambda}+o\left(\frac{1}{N}\right)
$$

or changing the variable $x$ to $v=\mathrm{i} u=x-\mathrm{i} \lambda / 2$

$$
\ln T(v)=-N f(v)+\frac{\pi \mathrm{i}}{6 N}(c-24 \Delta) \cosh \frac{\pi v}{\lambda}+o\left(\frac{1}{N}\right)
$$

where the central charge is

$$
c=1-\frac{6 \lambda^{2}}{\pi(\pi-\lambda)}
$$

and the conformal weights are

$$
\Delta=\frac{\phi^{2}-\lambda^{2}}{4 \pi(\pi-\lambda)}
$$

For the ground state, $s=t=1$ yields $\Delta=0$. The choice of $1<s \leq h-1$ and $1<t \leq h-k-1$ gives the excited states. Remarkably, inserting $\lambda$ given by (1.12) into the conformal spectra we have the central charges and the conformal weights of the primary fields for Virasoro minimal models

$$
\begin{gathered}
c=1-\frac{6 k^{2}}{h(h-k)} \quad \text { and } \quad \Delta=\frac{[h t-(h-k) s]^{2}-k^{2}}{4 h(h-k)} \\
k<h ; \quad s=1,2, \cdots, h-1 ; t=1,2, \cdots, h-k-1
\end{gathered}
$$

for $k \geq 1$. The unitary minimal models are given by taking $k=1$.

\section{Discussion}

In this paper we have obtained the conformal spectra of the non-unitary minimal conformal field theories from the finite-size corrections to the eigenvalue spectra of the transfer matrix of the critical ABF model on the regime III/IV critical line with the crossing parameter (1.12). The method given in [20] is only for calculating the central charges for the six-vertex model with a twisted boundary condition. In this paper it has been generalized to calculate 
both the central charges and the conformal weights. Other methods, for example, the thermodynamic Bethe ansatz (TBA) analysis (see [8], [28]- [35]), exist for calculating the conformal spectra. The TBA relies heavily on the string hypothesis, while our method crucially depends on the ANZ property instead. However, it is an interesting problem to generalize the TBA method for calculating the conformal weights of the ABF SOS model.

There is another method for calculating the finite-size corrections of transfer matrices. This has been shown by solving the fusion hierarchies of the ABF model. Unfortunately it is only for $k=1$ [11] (also see [21]). It has not yet known how to find finite-size corrections of the transfer matrix of the ABF model for $k>1$.

\section{Note Added}

After this work was submitted I was informed by Murray Batchelor about reference [36], where the authors generalize the method of [20] to calculate the conformal spectrum of the six-vertex model with twisted boundary conditions. However, the finite-size corrections to the transfer matrix of the ABF SOS model with the more general crossing parameter (1.12), which is an important class of integrable lattice models corresponding to realizations of non-unitarity minimal conformal field theories, was not considered there. I am grateful to Murray Batchelor for drawing my attention to [36].

\section{Acknowledgements}

This research has been supported by the Australian Research Council. The author also thanks P. A. Pearce and Ole Warnaar for discussions.

\section{References}

[1] G. E. Andrews, R. J. Baxter and P. J. Forrester, J. Stat. Phys. 35 (1984) 193.

[2] A. A. Belavin, A. M. Polyakov and A. B. Zamolodchikov, Nucl. Phys. B241 (1984) 333.

[3] D. A. Huse, Phys. Rev. B30 (1984) 3908.

[4] D. Friedan, Z. Qiu and S. Shenker, Phys. Rev. Lett. 52 (1984) 1575.

[5] I. Affleck Phys. Rev. Lett. 56 (1986) 746.

[6] A.N.Kirillov and N.Yu.Reshetikhin, J. Phys. A20 (1987) 1565;1587.

[7] R. J. Baxter, J. Stat. Phys. 28 (1982) 1.

[8] V. V. Bazhanov and N. Yu Reshetikhin, Int. J. Mod. Phys. B4 (1989) 115.

[9] A. Klümper and P. A. Pearce, J. Stat. Phys. 64 (1991) 13. 
[10] A. Kuniba and T. Nakanishi, "Spectra in Conformal Field Theories from the Rogers Dilogarithm" preprint 92.

[11] A. Klümper and P. A. Pearce, Physica A 183 (1992) 304.

[12] H. J. de Vega and F. Woynarovich, Nucl. Phys. B251 (1985) 439.

[13] F. Woynarovich, Phys. Rev. Lett. 59 (1987) 259.

[14] G. von Genlen and V. Rittenberg, J. Phys. A20 (1987) 227.

[15] H. J. de Vega and M. Karowski, Nucl. Phys. B285 (1987) 619.

[16] C. J. Hamer, G. R. W. Quispel and M. T. Batchelor, J. Phys. A20 (1987) 5677.

[17] M. Karowski, Nucl. Phys. B300 (1988) 473.

[18] F. C. Alcaraz, M. N. Barber and M. T. Batchelor, Phys. Rev. Lett. 58 (1987) 771.

[19] A. Cappell, C. Itzykson and J.-B. Zuber, Nucl. Phys. B280 (1987) 445; Commun. Math. Phys. 113 (1987) 1.

[20] A. Klümper, M. T. Batchelor and P. A. Pearce, J. Phys. A24 (1991) 3111.

[21] Y. K. Zhou and P. Pearce, Nucl. Phys. B (1995), in press.

[22] Y. K. Zhou, Nucl. Phys. B (1995), in press.

[23] P. J. Forrester and R. J. Baxter, J. Stat. Phys. 38 (1985) 435.

[24] C. N. Yang, Phys. Rev. Lett. 19 (1967) 1312.

[25] R. J. Baxter, Ann. Phys. 70 (1972) 193.

[26] L. A. Takhtadzhan and L. D. Faddeev, Russian Math. Survey 34:5(1979) 11.

[27] S. O. Warnaar, M. T. Batchelor and B. Nienhuis, J. Phys. A25 (1992) 3077.

[28] C. N. Yang and C. P. Yang, J. Math. Phys. 10 (1969) 1115.

[29] M. Takahashi, Prog. Theor. Phys. 46 (1971) 401.

[30] M. Takahashi and M. Suzuki, Prog. Theor. Phys. 48 (1972) 2187.

[31] Al. B. Zamolodchikov, Phys. Lett. B253 (1991) 391; Nucl. Phys. B358 (1991) 497.

[32] T. R. Klassen and E. Melzer, Nucl. Phys. B338 (1990) 485.

[33] T. R. Klassen and E. Melzer, Nucl. Phys. B350 (1991) 635.

[34] M. J. Martins, Phys. Rev. Lett. 67 (1991) 419.

[35] A. Kuniba, Nucl. Phys. B389 (1993) 209.

[36] A. Klümper, T. Wehner and J. Zittartz, J. Phys. A26 (1993) 2815. 